\title{
XXXIX.
}

\section{Ueber die Analyse der Legirungen von Kupfer und Zink.}

\author{
Von \\ Rivot und Bouquet.
}

(Annal. de Chimie et de Phys. XXXIIl., 24.)

Die Trennungsarten des Zinks und des Kupfers, welche in den besten analytischen Werken angeführt sind, beruhen auf der Anwendung des Schwefelwasserstoffes oder des Kalis. II. Rose empfiehlt das Schwefelwasserstoffgas und verwirft durchaus den Gebrauch des Kalis, da das Kupferoxyd stets eine gewisse Menge Zinkoxyd zurückhalte.

Noch erwähnen wir der zahlreichen Versuche von Pelouze; ${ }^{*}$ ) sie können als ein Muster von Genauigkeit, hinsichtlich der Bestimmung des Kupfers angesehen werden.

Das Verfahren von Pelouze besteht in der Fällung des Kupfers aus einer ammoniakalișchen Flüssigkeit, vermittclst einer titrirten Lösung des Natronsulphihydrats; aber das crhaltene Oxysulphuret des Kupfers Jässt sich in Folge der schnellen Oxydation bei der Berührung mit der Luft schwierig filtriren. Ferner ist es fast unumgänglich nöthig, das Zink durch Differenz zu bestimmen.

Da wir vom Versuchsbureau der Ecole des Mines beauftragt wurden, viele Messingsorten zu analysiren, so stellten wir Versuche über dic Trennung des Kupfers und des Zinks an, die uns überzeugt haben, dass Schwefelwasserstoff kein befriedigendes Resultat giebt.

Hat man eine schwach saure Flüssigkeit, so fällt Schwefelwasserstoff gleichzeitig mit dem Kupfer fast die ganze Menge des Zinks als Schwefelmetalle. Ist die Flüssigkeit stark sauer, so wird stets eine beträchtliche Menge Schwefelzink mit dem Schwefelkupfer niedergerissen, wie sauer auch die Flüssigkeit sein mag.

") d. J. 37., 440. und 38., 407. 
Wir suchten nach einer andern Trennungsweise und fanden, dass das Kali unter Anwendung gewisser Vorsichtsmaassregeln sehr genau das Kupferoxyd rom Zinkoxyd trennen kaun.

\section{Anwendung von Schwefelwasserstoff.}

Die Versuche, gewogene Mengen von Kupfer und Zink, miltelst Schwefelwasserstoff zu trennen, warden auf folgende Weise ausgeführt:

Beide Metalle werden in Salpetersüure gelüst, die Nitrate werden verdampft und in Oxyde verwandelt; die Oxyde lüst man in Chlorwasserstoffsäure; in die mehr oder weniger saure Lüsung leitet man einen Ueberschuss von Schwefelwasserstoff. Der Niellerschlag, welcher alles Kupfer und einen Theil Zink als Schwefelmetalle enthailt, wird auf einem Filter gesammelt und mit schwefelwasserstofthaltigem Wasser ausgewaschen. Nach dem Trocknen trennt man ihn vom Filter und lüst ihn in Künigswasser. Die Flüssigkeit wird ammoniakalisch gemacht, und das liupfer durch Kali als Oxyd gefällt. Zur Bestatigung reducirt man das hupferoxyd durch Wasserstoffyas und bestimint das Kupfer im metallischen Zustande.

Das Zink findet sich in zwei Flüssigkeiten; erstens in der mit Schwefelwasserstofl behandelten chlorwasserstoffsauren Flüssigkeit; ferner in der alkalischen Flüssigkeit, aus welcher das Kupfer durch liali getält worden ist.

In der ersten Flüssigkcit kann man die Menge des Zinks bestimmen, welche der Einwirkung des Schwefelwasserstolls entgangen ist, wemn man Ammoniak und Schwefelammonium hinzufügt. Das dadurch erhaltene Schwefelzink wird auf einem Filter gesammelt, sorgfältig getrocknet, gut rom Filter getrennt alsdann durch Rüsten und Glühen in Oxyd verwandelt.

Um das in der alkalischen Flüssigkeit enthaltene Zink zu bestimmen, muss man die Alkalien durch eine Säure sättigen und das Zink durch ein kohlensaures Alkali fällen. Der gut ausgewaschene und getrocknete Niederschlag wird durch Glühen in Oxyd verwandelt.

\section{Versuche.}

A. - Eine Legirung von Kupfer und Zink aus $\mathrm{Ca} 0,96 \mathrm{Grm}$.

Zn 1,04 ” 
wurde auf obige Weise behandelt, und die chlorwasserstoffsaure Flüssigkeit vor dem Einleiten von Schwefelwasserstoff hinreichend angesüuert, um das Lakmuspapier stark zu rüthen, olne aber die Menge der Säure zu bestimmen. Die Menge des nicht durch Schwefelwasserstoff gefïllten Zinks war in drei Versuchen

$\begin{array}{lll}\text { 1ster Versuch } & 0,055 \mathrm{Grm} . \\ \text { 2ter } " & 0,060 \quad " \\ \text { 3ter } ", & 0,080 \quad "\end{array}$

Diese Resultate craben die fast vollstündige Füllung des Zinks durch Schwefelwasserstoff; das Resultat wird bestätigt durch die Untersuchung ler alkalischen Flüssigkeit, welche nach der Fällung des Kupfers durch Kali übrig bleibt.

B. - Wir haben alsdann Flüssigkeiten von einem bestimmten Säuregchalt angewandt:

$$
\begin{array}{lll}
\text { Zn } & 0,82 & \text { Grm. } \\
\text { Cu } & 0,85 \quad,
\end{array}
$$

Die beiden Iletalle wurden in Oxyde verwandelt, alsdinn in einer Flüssiglkeit gelüst, welche enthielt:

Wasser $200 \mathrm{Grm}$.

Rauchende Chlorwasserstoffsäure 20

Durch Behandlung der Flüssigkeit mit Schwefelwasscrstoff und durch Bestimmung des nicht gefällten Zinkes, fanden wir: Zn 0,672 Grm.

Dies giebt für das gleichzeitig mit dem Kupfer präcipitirte Zink 0,148 Grm., also $18 \%$.

C. - Wir nahmen:

$$
\begin{aligned}
& \mathrm{Zn} 1,10 \mathrm{Grm} . \\
& \mathrm{Cu} 1,10,
\end{aligned}
$$

Die in Oxyde rerwandelten Hetalle wurden in eincr Flüssigkeit gelöst, welche bestand aus
Wasser $200 \mathrm{Grm}$.
Rauchender Chlorwasserstoffsäure 50 "

Nach dem beschriebenen Verfahren erhielten wir: Zink, nicht durch Schwefelwasserstoff gefiallt 1,016 Grm. *)

Zink, gefällt durch Schwefelwasserstoif $\mathbf{0 , 0 8 4 ~ " ~}$

*) In den Berechnungen ist das Aequivalent des Zinks 406 und dias des Kupfers 395. Wir haben uns von der Richtigkeit dieser Ictzten Zahl 
also 7,63\% durch Schwefelwasserstoff gefülltes Zink. Bei diesem Versuche ergab dic Bestimmung des Kupfers:

durch Kali gefälltes Kupferoxyd 1,39 Grm.

und wir erlielten durch Reduction des Kupferoxydes mit Wasserstoff:

\section{Metallisches Kupfer 1,098 Grm.}

Wir hahen uns überzeugt, dass nach der Füllung des Kupfers die alkalische Flüssigkeit eine sehr bemerkliche Menge von Zink enthielt.

D. - Bei der Operation mit einem Gemenge aus:

$$
\begin{array}{lll}
\mathrm{Cu} & 1,00 \mathrm{Grm} . \\
\mathrm{Zn} & \mathbf{0 , 5 4}
\end{array}
$$

und durch Aufösen der Oxyde in einer Flüssigkeit, welche wie im vorhergehenden Versuche aus

\section{Wasser} 200 Grm.

Rauchender Chlorwasserstoffsäure $\mathbf{5 0 ~ "}$

hestand, erhielten wir unter Anwendung von Schwefelwasserstoff:

$$
\begin{array}{ll}
\text { Nicht gefälltes Zink } & \mathbf{0 , 4 1 6} \text { Grm. } \\
\text { Gefälltes Zink } & \mathbf{0 , 1 2 4},
\end{array}
$$

demnach 22,90\% durch Schwefelwasserstoff gefälltes Zink.

Die Bestimmung des Kupfers ergab:

durch Kali gefälltes Kupferoxyd 1,250 Grm.

durch Reduction des 0xydes mit Wasserstoff erhaltenes Kupfer 0,995 Grm.

E. - Durch dasselbe Verfahren und mit demselben Verhältniss von Wasser und Chlorwasserstoffsäure, erhielten wir aus einem Gemisch von

$$
\begin{array}{ll}
\text { Cu } & 0,70 \\
\mathrm{Zn} & \mathbf{G r m} .20
\end{array}
$$

durch Schwefelwasserstoff nicht gefälttes Zink 1,088 Grm. durch dieses Reagens gefälles Zink 0,112, also 9,30\% gleichzeitig mit dem Kupfer durch Schwefelwasserstoff gefältes Zink.

mehrmals überzeugt, indem wir bestimmte Mengen ron sehr reinem metallischen Kupfer in 0xyd verwandelten, und daraus durch Wasserstoffgas das metallische Kupfer regenerirten. 
Die Bestimmung des Kupfers ergab uns:

Finpferoxyd $0,870 \mathrm{Grm}$.

Kupfer durch Reluction des 0xydes mittelst Wasserstoff 0,692 Grm.

F. - Wir hatten ein Gemisch von

Cu 1,00 Grm.

Zn 1,04 "

Die beiden in Oxyde verwandelten Metalle wurden in einer Flüssigkeit gelöst, welche enthielt:

Wasser

$125 \mathrm{Grm}$.

Rauchende Chlorwasserstofsäure $12 \check{5}$ "

Die Aullösung war demnach viel saurer als die gewöhnlich bei den Analysen angewandten Flüssigkeiten.

Der Schwefelwasserstoff fällte alles Kupfer, gleichzeitig aber eine gewisse Henge Zink; wir fanden

durch Schwefelwasserstoff nicht gefältes Zink 0,920 Grm.

durch dieses Reagens gefilltes Zink 0,120 " also $11,54 \%$ grleichzeitiy mit dem Kupfer gefältes Zink.

Diese Versuche scheinen uns gạnz deutlich zu beweisen, dass der Schwefelwasserstoff nicht allein zur Trenumg des Kupfers vom Zink angewandt werden kann. Wie sauer auch die Flüssigkeit, in die man Schwefelwasserstoff einleitet, sein mag, das Schwefelkupfer wird eine veränderliche, stets aber sehr beträchtliche Menge Zink mit niederreissen.

Ebenso folgt aus diesen Versuchen, dass, wenn man den durch Schwefelwasserstoff erhaltenen Niederschlag in Königswasser löst, und das Kupfer als Kupferoxyd durch Kali aus einer vorher ammoniakalisch gemachten Flüssigkeit fällt, man für die Bestimmung des Kupfers sehr genane Resultate erlangt.

\section{Anwendung des Kali.}

Nach diesen Versuchen wandten wir das Kali zur Trennung beider Metalle an. Diese Trennungsweise wurde übrigens von Vauquelin in einer Abhandlung über die Analyse des Messings (Annales de Chimie 1. Serie XXXIII) empfohlen.

Vauque lin empfieblt das Messing in Salzsāure zu lōsen, die Lösung in der Kälte und in einem verschlossenen Gefäss durch Kali zu fällen, die filtrirte Flüssigkeit mit Schwefelsãure zu sättigen und das Zink durch kohlensaures Kali nieder zu schlagen. 
Dieses Verfahren lieferte uns sehr gute Resultate; wir fanden es aber für vortheilhaft, das Kupferoxyd aus einer ammoniakalisch gemachten Flüssigkeit durch Kali zu fällen. Das erhaltne Kupferoxyd hält kein Zinkoxyd zurück, ebenso wenig enthailt es Kali, während, wenn man eine nicht ammoniakalische Flussigkeit anwendet, es fast unmöglich ist, dem Kupferoxyd selbst durch lang anhaltendes Waschen mit kochendem Wasser alles Kali zu entziehen.

Die Fällung des Kupferoxyds durch Kali in einer ammoniakalischen Flüssigkeit geschielt sehr gut bei einer Temperatur von 76 bis $80^{\circ}$; bei einer höhern Temperatur haftet Kupferoxyd so fest den Wänden des Gefüsses an, dass man genöthigt ist es, wiederum in einer Sãure zu lösen und von Neuem za fällen.

Es ist nothwendig, nur verdünnte l'lüssigkeiten und keinen zu grossen Ueberschuss von Kali anzuwenden. In concentrirten Flüssigkeiten oder bei einem Ueberschuss von Alkali erhält man cin Kupferoxyd, welche hartnäckig eine ansehnliche Menge Kali zurückhält.

Ausführung der Analyse. - Wir verfalıren bei der Analyse einer Legirung von Kupfer und Zink auf folgende Weise:

Wir lüsen die Legirung in Salpetersäure, verdünnen mit Wasser und sättigen die Säure mit Ammoniak; der ammoniakalischen Flüssigkeit fügen wir einen kleinen Ueberschuss von reinem Kali in Stücken hinzu, und erhitzen gelinde in Sandbade bis die Flüssigkeit vollständig enträrbt ist und nicht mehr nach Ammoniak riecht. Wir filtriren und waschen das Kupferoxyd mit kochendem Wasser aus.

Zu der alkalischen Flüssigkeit setzen wir Chlorwasserstoffsäure bis eine saure Reaction eintritt, alsdann fällen wir das' Zink durch kohlensaures Natron. Vor dem Filtriren erhitzen wir 7 bis 8 Stunden im Sandbad, um die freie Kohlensāure zu verjagen. Der alsdann filtrirte Niederschlag wird mit kochendem Wasser ausgewaschen, getrocknet, vom Filter getrennt und geglüht.

Dic beiden Metalle werden demnach als Oxyde bestimmt. Nach diesem Verfahren analysirten wir zwei Mischungen:

und wir erhielten:

$$
\begin{array}{lll}
\text { Cu } 1,00 \mathrm{Grm} . & 0,70 \mathrm{Grm} . \\
\mathrm{Zn} & 1,00 ., & 1,10 "
\end{array}
$$




\section{Kupferoxyd 1,25 Grm. $\quad 0,870 \mathrm{Grm}$. Zinkoxyd 1,260 " 1,350 ,} entsprechend 1,008 Grm. und 1,08 Grm. Zink.

Das Kupferoxyd wurde durch Wasserstoffgas reducirt, und wir erhielten:

Metallisches Kupfer 0,997 Grm. 0,692 Grm.

Ist das Verhältniss des Zinks sehr gross, so wendet man zur leichteren Trennung zuerst Schwefelwasserstoff an, löst dann das gefällte Schwefelmetall nochmals in Königswasser, und fällt aus der ammoniakalisch gemachten Lösung das Kupfer durch Kali. Die Bestimmung des Kupfers ist dann sehr genau, die des Zinks etwas weniger genan, da es in zwei Theile getrennt wurde. Man könnte zwar die beillen Zink enthaltenden Flüssigrlseiten vereinigen, nachdem aus der einen durch Wärme der Schwefelwasserstoff verjagt, und die andre angesäuert worden ist, um das Zink auf einmal zu fïllen. Allein in einer so zusammengesetzten Flüssigkeit, dic eine gewisse Menge eines Kalisalzes enthail, kann man nicht das Zink als Schivefelmetall zu fällen suchen. Die schnelle Oxydation, welches dieses Sulphür bei der Berührung der Luft erleidet, erlaubt nicht ein fortgesetztes Auswaschen, was doch zur vollstïndigen Entfernung alles Kalisalzes nüthig ist.

Man muss demnach das kollensaure Natron anwenden und der Niederschlag wird in diesem Falle um so schwieriger auszuwaschen sein, je grüsser seine Henge ist. Wir haben ziemlich befriedigende Resultate erhalten, wenn wir die Bestimmung des Zinks in zwei Theilen vornalımen.

So haben wir bei den oben erwähnten Versuchen D, E, F, wo wir folgende Mengen von Zink in Arbeit nahmen:

cinerseits erhalten:

$0,54 \mathrm{Grm}$. $\quad 1,20 \mathrm{Grm}$. $1,04 \mathrm{Grm}$.

$$
\text { 0,416 Grm. } \quad 1,088 \mathrm{Grm} \text {. } \quad 0,920 \mathrm{Grm} \text {. }
$$

durch Schwefelwasserstoff nicht gefälltes Zink, welches aus der chlorwasserstoffsauren Lüsung mit Schwefelammonium gefüllt und dann als 0xyd gewogen wurde, ferner
$0,117 \mathrm{Grm}$.
$0,109 \mathrm{Grm}$.
$0,115 \mathrm{Grm}$.

Zink, welches aus der angesäuerten haliftüssigkeit durch kohlensaures Natron gefällt und ebenfalls als 0xyd gewogen worden war. 
Die Gesammtmenge des erhaltenen Zinks giebt in den drei Versuchen folgende Zahlen:

$$
\text { 0,523 Grm. 1,197 Grm. 1,03j Grm., }
$$

welche sich hinreichend den angewandten Mengen von Zink nähern.

Die so eben mitgctheilten Resultate scheinen uns ziemlich befriedigend den Beweis zu liefern, dass die Trennung des Zinks und des Kupfers recht gut gelingt bei Anwendung von Kali oder auch von Schwefelwasserstoff und Kali.

Schwefelwasscrstoff allein angewendet, liefert stets sehr ungenaue Resultate.

Uebrigens veranlasst der Schwefelwasserstolf, als Trennungsmittel der Hetalle in sauren Flüssigkeiten läufig ungenügende Resultate. So kann dieses lieagens nicht zur Scheidung des Bleis vam Zink angewandt werden; es giebt sugar noch ungenauere Resultate als bei der Trennung des Kupfers vom Zink. Der Grund davon ist sehr einfach: Das Kupfer lässt sich durch Schwefelwasserstoll in einer stark chlorwasserstoffsauren Flüssigkeit fällen, während die Füllung des Bleis nur gut gelingt in ciner verdünnten und wenig sauren Flüssigkeit; in diesem Falle wird sich aber fast die ganze Menge des Zinks gleichzeitig mit tem Blei niederschlagen. Wir hatten im Laboratorium der École des Mines mehrere Beispiele der vollständigen Fällung des Zinks bei den Analysen der Blende und bleiglanzhaltigen Mineralien, in denen über 50 p. C. Blende enthalten war.

Auch bei den Analysen der nickel- und kupferhaltigen Legirungen fält Schwefelwasserstoff, wenn er zur Trennung der beiden Hetalle angewendet wird, mit dem Kupfer stets noch eine betrüchtliche Menge Nickel, wenn auch die chlorwasserstullsaure Lösung der beiden Netalle stark sauer ist.

Wir werden jetzt nicht weitliuliger auf diesen Gegenstand eingehen, wir behalten uns aber vor, später die Resultate unserer Versuche mitzutheilen, die wir hinsichtlich der Anwendung des Schwefelwasserstoffes bei Analysen unternommen haben. 